\title{
Can Internalism and Externalism be Reconciled in a Biological Epistemology of Language?
}

\author{
Prakash Mondal
}

Received: 19 January 2011 / Accepted: 2 May 2011

(C) Springer Science+Business Media B.V. 2011

\begin{abstract}
This paper is an attempt at exploring the possibility of reconciling the two interpretations of biolinguistics which have been recently projected by Koster (Biolinguistics 3(1):61-92, 2009). The two interpretations - trivial and nontrivial - can be roughly construed as non-internalist and internalist conceptions of biolinguistics respectively. The internalist approach boils down to a conception of language where language as a mental grammar in the form of I-language grows and functions like a biological organ. On the other hand, under such a construal consistent with Koster's (Biolinguistics 3(1):61-92, 2009), the non-internalist version does not necessarily have to be externalist in nature; rather it is a matter of mutual reinforcement of biology and culture under the rubric of a co-evolutionary dynamics. Here it will be argued that the apparent dichotomy between these two conceptions of biolinguistics can perhaps be resolved if we have a richer synthesis that accounts for both internalism and non-internalism.
\end{abstract}

Keywords Biolinguistics · Internalism · Non-internalism · Theoretical synthesis

\section{Introduction}

Recently Koster (2009) has argued in favor of a conception of language within which language can be seen as a cultural phenomenon having a socio-cultural reality but ultimately grounded in human biology. Under such an interpretation Koster's (2009) version of biolinguistics is 'trivial' in nature. On the other hand, the internalist conception of language where language is viewed as a mental grammar in the form of I-language that grows and functions like a biological organ has been rejected by Koster (2009). This has been called the 'non-trivial' version of biolinguistics in that such a concept of language is narrowly construed to fit the

P. Mondal $(\bowtie)$

Indian Institute of Technology, Delhi, India

e-mail: prakash.mondal@research.iiit.ac.in 
characterization of language faculty as some 'core capacity' which is constituted by recursion. The entire issue has repercussions and implications centered around the debate on the dichotomy between internalism and externalism recently revived by Lohndal and Narita (2009), who advocate the methodology of internalism as championed by Chomsky $(1986,1995,2000)$. Overall, it seems that the dichotomy is something that can never be resolved at all. As Koster (2009) traces the history of the debate, it becomes clear that the idea of language as being external to the individual was advocated by linguists of the first half of 20th century and even goes back to Von Humboldt. With Chomskyan revolution in linguistics starting in the 1950 s, the concept of internalism came up and began to be firmly rooted in scientific theories of language that were developing. And the current situation is such that the internalist vs. externalist debate goes on in the guise of autonomy vs. non-autonomy or modularity vs. distribularity or even biology vs. culture from diverse perspectives and at different scales in fields ranging from theoretical linguistics, sociolinguistics, anthropology, psycholinguistics, psychology, cognitive neuroscience, evolutionary biology, artificial intelligence to perhaps molecular genetics. The aim is not to resolve all these dichotomies in one go; rather it will be argued that all these dichotomies exhibit some central tendencies and patterns which can be addressed best if they are couched in terms amenable to a better understanding of the path toward a resolution. The nature of the stalemate is such that specifically within the discipline of linguistics, both internalist theories like generative grammar and externalist theories like functional grammar (Givón 1995, 2002, 2005; Dik 1997a, b; Hengeveld 2005) or systemic functional grammar (Halliday 1973, 1985) co-exist. The field of sociolinguistics is also externalist in nature.

The purpose of this paper is to have a refreshingly new look at the entire issue in order to reformulate the debate in a manner that may show a path toward resolving the stalemate. It will be hypothesized that intrinsic nature of language shows complementarity between internalist state and externalist state in that it shifts, undulates (it will be explicated in later sections) between two modes of existence in an elastic manner. It is as if knowledge of language is like an elastic sheet shifting and undulating continuously between two forms of existence. In other words, this means that language functions both as an internalized form of mental grammar in individual brains and as an externalized socio-cultural object existing at an intersubjective plane of reality by shifting between these two poles of reality. Hence a truly unified biolinguistic approach toward language should aim at accounting for such a complementarity. Even if such a synthesis is unlikely to be fully fleshed out at this stage, an outline of such a proposal specifying the skeletal rudiments will be drawn up here with some tentative and speculative links to lines of research in cognitive psychology, evolutionary biology and theoretical linguistics.

\section{Internalism vs. Externalism}

The dichotomy between internalism and externalism is a jumping off point for any discussion that is pitched at tearing apart the connection between brain-internal or mind-internal operations and brain/mind-external objects like lexicon of a language. Koster (2009) rightly argues that before making such a distinction we must be able 
to tell apart what is internal to mind/brain from what is external to it. Given that the boundaries of the brain stop at the cranium, the boundaries of mind are porous and fluid (Clark 1997). This makes the notion of mind-externality or mind-internality problematic. In the same manner the notion of mentally internalized grammar also becomes problematic. This is the reason why Koster (2009) has vouched for a kind of externalism which is, in fact, non-internalism that is ultimately rooted in internalism which, if correctly construed, equates to biologically instantiated generative system of language responsible for unlimited amount of linguistic expressions as has been argued by Lohndal and Narita (2009). Clarifying the nature of internalist methodology, Lohndal and Narita (2009) have, on the other hand, argued that internalism as construed in the sense above does not deny externalism, but it leaves it beyond the object of scientific study of language. That is why Chomsky's notion of E-language was developed. If we do not, for the time being, go into Burge or Putnam type of meaning externalism, both Koster's (2009) externalism and Chomsky's internalism converge on a common ground. Koster's (2009) externalism is rooted in Chomskyan internalism and Chomskyan internalism does not deny facts about the supra-individual nature of language. Koster's 'trivial' version of biolinguistics morphs into a kind of non-internalism because under such a construal, language is fundamentally a sociocultural phenomenon or entity which happens to be grounded in biology just like other sociocultural entities. Interestingly, Carr (1990) has already tried to strike a balance between extra-individual linguistic objects and brain internal operations in the form of 'interactionism', even if he has not clearly articulated his own form of 'interactionism'. So the concept of externalism as can be applied here extrapolates Carr's notion of linguistic objects being extra-individual to the whole of linguistic knowledge when linguistic knowledge as a whole can be seen as externalized. Such a view of externalism considers processing mechanisms to be brain-internal but the linguistic objects to be intersubjective and supra-individual. This issue will be touched upon below at far greater depths.

In a nutshell, it appears to be the case that internalism vs. externalism dichotomy ceases to exist once they are shown to be not alternatives to each other, but complementary to each other. Even each side of the debate acknowledges the existence and contribution of the other. It can be noted that external objects cannot exist on their own except thanks to our internal biological capacities; internal biological capacities, on the other hand, cannot grow, develop and function unless grounded in the external world. Both Koster (2009) and Lohndal and Narita (2009) seem to agree on this. Building on such insights, we can now clearly articulate and formulate the outline of a proposal toward a reconciliation that incorporates both internalism and externalism by accounting for both at some level. In doing this, issues of similar magnitude and nature like modularity vs. distribularity or autonomy vs. nonautonomy will also be handled, as they verge on similar principles on which internalism vs. externalism dichotomy is based. What is being postulated is that the complementarity existing at the level of language vis-à-vis the world out there parallels the complementarity at the intra-linguistic level among the components of language like syntax, semantics, and phonology. So along these lines, it is hypothesized that complementarity between modularity and distribularity/interactionsism at the intra-linguistic level possibly goes all the way up to language-world 
relations as a whole when language shifts between internalized knowledge and externalized public extension.

For this purpose, basic elements of a new architecture of language will first be drawn up. The internal form of the architecture will be formulated in a manner such that it can account for the complementarity between modularity or autonomy of the components of language on one hand, and the distribularity/interactionsim among them on the other. And then it will be shown how such an architecture shows patterns of complementarity between individualized knowledge and its public extension. The broader outline of such an architecture will perhaps help us closely approximate a united conceptualization of language where biology of language and its epistemological nature will be intertwined.

\section{A General Architecture of Language}

Here a general functional architecture of language will first be presented with an eye on its potential implications for the overall framework that outlines a unified biolinguistic conceptualization of language to be explored here. A certain generalizations will be drawn up from this general architecture of language to be implemented in and intertwined with the underlying cognitive substrate. Such implications will be extrapolated from the internal form of the architecture of language showing complementarity both below and above. Let's see how we can approach it. A range of studies in linguistic phenomena (Neelman and Weerman 1998; Hengeveld et al. 2004; Mascaró 2007) are increasingly pointing toward more interconnected, fluid interactions among the components of language- syntax, semantics, lexicon, morphology and phonology.

The architecture in Fig. 1 is a functional (computational) level elastic architecture of language in mind/brain (Mondal 2009). The nodes are functional modules, not in Fodorian sense. They are functionally coherent units of representational resources realized in diffuse, often shared networks of brain regions (Gobet 2005; Downing et al. 2005). And the bi-directional arrows regiment processing connections among them. Here we get in the architecture processing of representations, so processing is unified with representations. Processing interacts with and influences the representations in $\mathrm{mind} /$ brain in a mutual modulatory dynamics so that representations in mind/brain are influenced by aspects of processing and vice versa (Hawkins 2004). Hence, processing applied to representations will constitute (linguistic) structures which include derivations (possibly in the form of Merge and Copy operations if insights from generative grammar are drawn).

In the proposed architecture above computation can run in any direction involving any number of language domains or components of language like syntax, semantics,

Fig. 1 A multipartite unified functional architecture of language

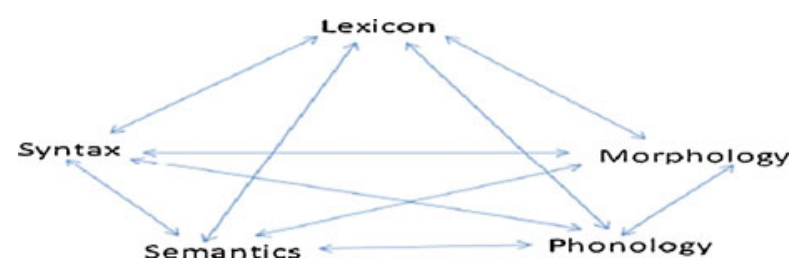


morphology etc. for the interpretation of any type of interaction. Different linguistic phenomena will involve different sets of interactions among the components. The hallmark of this architecture is its flexibility or elasticity; so the architecture can contract into a one-domain interfacing configuration or into a two domain or threedomain interfacing configuration (and so on), and also expand into a two-domain or three-domain or four-domain interfacing configuration (and so on). Here interface effects involving the components/domains of language can be computed in parallel from and in any direction involving any number of domains or modules of language given that only some content of each as required by others is available to the others. This is ensured by a number of constraints operating over the architecture. The constraints also make sure that the architecture does not grow too powerful in being able to run computations in any direction involving any number of components of language. In fact, it is these constraints that give the architecture its elasticity. They are like pressures that warp the topological geometry of the architecture. It may well be argued that they are actually epigenetically encoded in our language faculty, in a form of closer but complex coupling between genes, and external as well as internal environment (Fitch 2009). In the next section they will be explored in details. However, here is a stipulation in order for the (algorithmic) computations in the architecture above. This can be stated as

Stipulation: For any linguistic phenomenon $\chi$ in any language $L$ there must be an interaction $\mathrm{I}(\chi)$ such that there is a set $\Omega$ of components of language which are in parallel interaction with each other with $\tau(\mathrm{I}(\chi))$ where the computational relevance is $\tau$ and $|\Omega|=\Theta$ and $\Theta \geq 1$ and $\Omega \neq \varnothing$ iff $I(\chi)$ satisfies a set $\Phi$ of constraints that operate on $\mathrm{I}(\chi)$.

Before we proceed, let's specify a number of concepts to be used for the formalizations in later sections below. A linguistic phenomenon is any phenomenon that involves a kind of interaction between any number of components of language for the observed regularities/irregularities found across languages. An interaction between any number of components of language involves a sort of interfacing between them such that such interfacing causes the components of language involved in the interaction to map required information onto each other, for example, the linguistic phenomenon of split intransitivity - a distinction between ergativity/ unaccusativity - causes syntax, lexicon, morphology and semantics to work in tandem and have impacts on one another. Computational relevance requires the mapping of as much linguistic information at the interfaces between the components of language as is feasible and needed in a given linguistic phenomenon. Adequacy of information is the amount of linguistic information as demanded in the requirements of computational relevance, so for example, in the linguistic phenomenon of middle constructions information about word order/linearity, agreement from syntax is to be characterized as adequate information.

\section{The Nature of $\Phi-$ Constraints on the General Architecture of Language}

As posited above, a range of constraints modulate the interactive dynamics of the linguistic domains or components in Fig. 1. Interaction between linguistic domains is 
such that some information in one domain is not accessible to the other domain(s). For instance, syllabification which is a matter of phonology is not of concern to syntax (Jackendoff 2002). Here are some more:

- Just as syllabification which is a matter of phonology is not of concern to syntax, generally it is not of concern to semantics as well.

- Multiple embedding in syntax is not relevant to phonology which is concerned about segments, suprasegments, syllable structure etc.

- Hierarchical organization of syntactic structures is not accessible to phonology which has nothing to do with how hierarchical organization affects the geometry of structures in syntax. So are perhaps features like locality, recursion in syntax which are not obtrusive to phonology.

- Let's now consider the cases below:

(i) The man left

(ii) The man was at home. He left in the evening.

In such cases as Gillon (2008) has shown, the internal argument can be implicit, so dropped. It is a matter of semantics as he argues as this falls under the domain of invariant features of lexicon and grammar. Hence it implies that this involves lexicon and syntax as well. But this does not have anything to do with phonology per se in that the internal structure of phonology does not bother about whether an argument is dropped under some interpretative conditions of semantics or not.

- Matters of quantification, variable binding in semantics also generally have nothing to do with phonology and little to do with lexicon, but of course a lot to do with syntax and semantics.

- There is also some information that is not visible to syntax from semantics or vice versa as well. For example, cases like ambiguity, vagueness have nothing to do with syntactic organization. That means syntax does not always determine how ambiguity is formed in sentences or how vagueness arises in syntactic structures. This information is certainly mediated by syntax which organizes the lexical items in a hierarchical format, but it does not have access to how vagueness and ambiguity emerge out of those structures. Had it been possible, we would not have found a difference in the following pair of sentences:

(iii) Every man loves a woman.

(iv) Every hunter kills a buck.

Clearly here the sentence (iii) is ambiguous with two scopal readings (one where men vary with respect to a single lady, and another where women vary with respect to different men) but (iv) is not. Has it anything to do with the syntactic structure? Lexicon and semantics and our world knowledge seem to be involved in that.

- In many cases it becomes very difficult to tell syntax apart from lexicon or morphology (Jackendoff 2007). The boundaries between them become fluid. Even if in Fig. 1 above five components of language have been drawn as being distinct, there will be specific constraints that may well determine when syntax and say, lexicon become indistinct- they may overlap with one another (as in cases like idiomatic expressions such as 'kick the bucket', 'take (something) for 
granted' which perhaps belong in both lexicon and syntax). Since the architecture in Fig. 1 is elastic enough, such fluidity is not banned when languages seem to demand that.

All this seems to point to the direction that there are differential ways in which different constraints affect the coordinated interactions among domains or components of language and one constraint may inhibit interaction between two domains, but enhances or stimulates interaction among others, often involving one of those two as well. For example, the case with (iii-iv) shows that even if interaction between syntax and semantics gets blocked by the constraint, but simultaneously it facilitates interaction between semantics and lexicon. So it seems that there is a sort of one-to-many mappings between constraints and the linguistic components or domains; it can be shown through the following:

$$
\mathrm{C}_{\mathrm{i}}\left(\mathrm{D}_{1}\right)=\xi_{1}, \mathrm{C}_{\mathrm{i}}\left(\mathrm{D}_{2}\right)=\xi_{2}, \mathrm{C}_{\mathrm{i}}\left(\mathrm{D}_{3}\right)=\xi_{3}, \ldots, \mathrm{C}_{\mathrm{i}}\left(\mathrm{D}_{\mathrm{n}}\right)=\xi_{\mathrm{n}}
$$

Here $C_{i}$ denotes any specific constraint, $D_{1}, D_{2}, D_{3}, \ldots, D_{n}$ are linguistic domains or components, and $\xi_{1}, \xi_{2}, \xi_{3}, \ldots, \xi_{n}$ denote specific effects of interactive dynamics among the linguistic domains.

Why the constraints operate in such a manner may have to do with evolutionary design of the language faculty. For this we may have to look at what these constraints reveal about language evolution. If not all information passes from one domain to another, it is most possibly because of the computational efficiency of processing. If all possible conditions applicable in one domain exhausting all information available in that domain get transferred to another domain, then there will be a computational overload or burden on the overall processing dynamics among the linguistic domains or components. And at the same time it is also true that the overall behavioral outcome of system depends on the components that constitute that system. If linguistic system is of such a nature, it will also show such a behavior. Now the question is: how constrained is it? This question is difficult to answer for all we know about the linguistic domains independently. Below the architectural constraints as imposed on the architecture in Fig. 1 will be shown to have significant amplifications and ramifications for the issue of reconciliation between internalism and externalism.

\section{Complementarity Between Modularity and Distribularity}

Significantly, the constraints of the kind above are neither exclusively absolute nor exclusively relative. A few are absolute and some are relative, needed only for a range of specific linguistic phenomena in different languages. Such constraints may well be the tools available in a toolkit version of Universal Grammar (Jackendoff 2002). They are like 'attractors' among which children acquiring natural language tend to choose from. As specified above, it is such constraints which warp the topological geometry of the architecture into a number of configurations which allow for one-component or two-component or threecomponent interfacing states and so on. If this is the case, modularity or autonomy of different components of language like syntax, morphology or phonology is not 
out of the ordinary. When the architecture warps into a one-component configuration where connections to all other components get blocked because of such warping, the architecture starts showing autonomy or modularity of that specific component, and in such cases other components of language do not stop functioning. They work in the manner which is imposed upon them by the very warping that causes the modularity or autonomy of the specific component of language. So what this entails is that all cases of modularity or autonomy do impact the functioning of the other components of language. If for example, we demand modularity of syntax, as generative grammar has done, other components of language like phonology, semantics will function in a way that is only by virtue of the operations performed by the component which has turned modular. In fact, this is what Minimalist Program (Chomsky 1995) has shown. Syntactic operations like Merge and Move starting with items drawn from Numeration are interpreted at interfaces PF (Phonological Form) and LF (Logical Form) after Spell-Out. This is what is described as the faculty of language in the narrow sense (FLN) by Hauser et al. (2002). And this is modular and autonomous. This is what the architecture in Fig. 1 turns into when specific constraints warp the architecture into modular configurations. Theoretical linguists have also argued for modularity of other components of language than syntax (Archangeli and Pulleyblank 1994; Di Sciullo $2005 \mathrm{a}, \mathrm{b})$. For that, the similar conditions may arise.

Now the architecture shows modularity or autonomy. What about non-autonomy or distribularity? Even if the term was actually coined by Barbara Finlay (see Kingsbury and Finlay 2001 for a fuller discussion) for an intermediate form processing between the fully distributed (as connectionists argue) and the fully modular (Fodor 1983), this term may refer to, if carried over to the architecture of language in Fig. 1 above, degrees of interaction spread out across the components of language. Such a case poses no problem for the architecture above. Weakening or slackening of certain constraints that trigger modularity can warp the architecture into an interfacing configuration where interaction flows in all directions among the components of language, thereby making the interaction among the components of language distributed in different degrees. The architecture under such circumstances ceases to be modular and starts showing patterns of distribularity. This is how the same architecture shifts, switches between complementary patterns of modularity and distribularity. This is in many ways similar to Kelso's concept of metastability which refers to a complementary pattern of stability and instability at the same time in coordination dynamics in a neural state-space (Kelso 1995, 2002; Kelso and Engstrom 2006).

But the question that naturally arises is: why do certain constraints cause modularity or distribularity by being reinforced or slackened? There are three reasons.

I. As already said above, constraints affect the coordinated interactions among the components of language and one constraint may inhibit interaction between two components, but enhances interaction among others. This may have perturbations in such a way that one component or domain of language gets segregated from others when the warping of the architecture triggered by the constraint apportions interactions of the other components away from the segregated component of language. 
II. The constraints operating over the general architecture of language are neither absolute nor relative. Some are absolute and some are relative to specific linguistic phenomena in specific languages. More specifically, the constraints constitute the borderlines of how much information one module or component of language can pass to the others as they are epigeneticallyrather than genetically-encoded in our language faculty. As development occurs, such constraints set out and fix the demarcations between the linguistic modules or domains. But throughout the lifetime of individuals, such constraints as specified show a tendency of being modified and changed as the research in developmental genetics and developmental neuroscience shows (Karmiloff-Smith 1992, 1998, 2006; Bates 1994, 1997, 1999; Dabrowska 2004; Marcus 2004; Johnson and Morton 1991; Johnson et al. 1993, 2009; Fletcher and Miller 2005; Diamond 2002, 2007, 2009). What follows from this is that the constraints that warp the geometry of the architecture of language are always continually changing; they are dynamical in nature throughout ontogenetic and perhaps phylogenetic time scales. This paves the way for a varying strength of the constraints. From an evolutionary point of view, it can be noted at this point that the constraints on the general architecture of language as specified above are encoded through a complex developmental process in which genes, complex mechanisms of cellular communication, socio-cultural factors, symbolic culture and other environmental factors etc. have equal causal roles if we follow the kind of Evo-Devo framework proposed by Balari and Lorenzo (2009). And it will be hypothesized that this is indeed highly possible in that development of those constraints at the individual level may have had enormous evolutionary significance by introducing novelties, major perturbations etc. Development of such constraints which may well constitute the cognitive phenotypes of language is non-linearly related to a set of morphogenetic and cognitive parameters which consist of genes, complex mechanisms of cellular communication, socio-cultural factors, symbolic culture, language use, language acquisition (or learning), evolutionary lineages all of which are evolutionarily significant. Hence, any change in any of these parameters may have had no impact on the development of those constraints across the evolutionary timescale.

III. The exact number of constraints operative over the architecture of language is not known. And this will be one of the greatest challenges ahead for future research into how many constraints operate on an otherwise stable architecture of language. For the time being, this has the implication that if we are not still aware of all constraints that operate on a general architecture of language, it is possible that some unknown constraints may well cancel out or strengthen the existing constraints as we know. Thus for example, a constraint that ensures that things like vagueness or ambiguity (which is a matter of importance to semantics) have nothing to do with hierarchical syntactic organization makes it possible for syntax to get segregated from semantics. Now suppose that there is/are some constraint(s) that we do not know of, but which may further block the transfer of information between semantics and other domains or components of language (apart from syntax). This may have the snowballing 
effect that semantics may ultimately get segregated from other components of language. So semantics does not care about what is happening elsewhere. The reverse scenario may make for cancelling out of the constraint above.

In sum, the continual shift between two complementarity states of modularity and distribularity is, to a large measure, similar in nature to the shift between internalized knowledge of language and its public extension. But the latter sort of complementarity is also closely tied to the constraints that operate over the general architecture of language. It is because it is such constraints which are individual in a strict sense; they tend to organize public forms of linguistic knowledge including perhaps E-language into I-language. This line of reasoning will be employed below to show how the constraints operating over the general architecture of language correspond to the organizing factors that work from outside to go inside the mind/brain by transforming, molding public forms of linguistic knowledge into internalized form of language.

\section{Complementarity Between Internalized Knowledge of Language and Externalized Knowledge of Language}

Now we can look into linguistic reality in Carr's (1990) sense in a totally different way. What is being proposed is that linguistic reality- whatever that turns out to beis not actually polarized, fixed, and unchanging. It always shifts, switches between two patterns of organization- internalized knowledge of language or I-language, and supra-individual intersubjective knowledge of language which consists of the lexicon of a language, the set of all rules of grammar of the language including semantics, morphology and phonology. As Carr (1990: 42) has argued it is never necessary that such externalized intersubjective or 'speaker-external' knowledge of language is isomorphous with individualized I-language. They are different yet overlapping organizations of knowledge. I-language must be a subset of the superset of such speaker-external knowledge of language. But the point to drive home is that it is quite clear that they are different poles of organization of epistemological reality. This is what has divided philosophers and linguists over whether language belongs to private or social-cultural reality. That is why we are now face to face with the dichotomy of internalism and externalism. Hence the need for a synthesis.

Koster (2009: 67) is right on a number of points as he reconstructs internalism and externalism. Processing and interpretation may well be private, but the notion of mind is not limited to only processing and interpretation. So he says

"Our cultural memory is stored in, and distributed over brains, including my own, and over libraries and other collections of media. The same is true for words and other linguistic expressions. It would be absurd to say that I remain within the confinements of I-language when I produce or understand a sentence exclusively with words from my own memory, but that I embark on a short excursion to E-language if I use a dictionary for one word or another in the middle of a sentence."

But Koster (2009) seems to be unclear as to how his non-internalism or externalism can exist only by virtue of internalism. Lohndal and Narita (2009: 325) 
are quite unequivocal on this point. They argue that it is by virtue of internalized knowledge of language that we can extrapolate externalized knowledge of language. The point can be made much clearer by fleshing it out the following way. What may be called intersubjective state exists only by virtue of there being individual subjective states. In a similar manner if we are committed to a form of internalism about the faculty of language, it is only by virtue of that internalized knowledge that we can make any claim regarding externalized knowledge. Here is an analogy. Population level concepts (in economics) like propensity to consume can only exist if there are individuals who consume food etc. If there is no individual possessing an internalized form of knowledge of language, there cannot exist any language at all. A warning should be made at this point. In no way is it being claimed that the architecture above in Fig. 1 is isomorphous with I-language as we know it. Whatever the form of I-language or FLN turns out to be in actuality, it is actually one of the many configuration states of the elastic architecture of language as specified in Fig. 1 above. But as long as it is instantiated in individual brains, it must be in some form of internalized knowledge. This internalized knowledge may or may not be isomorphous with I-language in a strictly Chomskyan sense. Let's call the first I'language as distinct from I-language, just to avoid misunderstanding.

Importantly, it may be possible that we are all looking in the wrong direction. Maybe it is just because of mind's computations being extended and distributed, we find a complementarity between internalized knowledge and externalized knowledge. It may sound really counter-intuitive and it may seem to be (as it does to Koster (2009)) "... absurd to say that I remain within the confinements of I-language when I produce or understand a sentence exclusively with words from my own memory, but that I embark on a short excursion to E-language if I use a dictionary for one word or another in the middle of a sentence". What if this is just what happens because of the very nature of our fluid mind? It is never being suggested that this is exactly what happens in reality. But maybe this is the missing link.

Here it is being proposed that externalized speaker-external intersubjective knowledge of language exists, and it remains disorganized until and unless it is organized into individual forms of I'-language by means of individual constraints that operate over the architecture of language which may well correspond to a basic format of our language faculty. Both forms of knowledge are real. It is being claimed that the externalized speaker-external knowledge of language exists, and it remains disorganized; we may, of course, try to organize, with our collective efforts, that intersubjective speaker-external knowledge of language into dictionaries, grammar books. But no dictionary can ever claim to have covered the entire lexicon of a language, nor can a grammar book ever cover the set of all rules of a language. So this externalized knowledge always remains unordered and disorganized. It is we who organize and order such knowledge into individualized chunks of I'-linguistic knowledge with the help of epigenetically encoded linguistic constraints which correspond to the constraints operating over a general architecture of language as described above. This is how we-or more specifically-epigenetically encoded linguistic constraints work outside-in by transforming, molding public forms of linguistic knowledge into internalized forms of language. Thus supra-individual or inter-individual forms of linguistic knowledge are transduced and molded into internalized forms of language thereby deriving internalism from externalism, in 
some sense. A similar view has been proposed by Barbieri (2010) who argues that the brain-wiring rules of the cognitive system underlying language (which is relatively new on the evolutionary trajectory) are provided by the community rules of interacting individuals who use and communicate in language. This is nothing other than an outside-in projection of community knowledge into individual brains. There is a continual shift between these two forms of knowledge of language as and when people use language. The case of a child acquiring a language can be considered here. The child being exposed to an externalized communal knowledge of language as belonging to a socio-cultural reality gradually organizes that knowledge into its own form of I'-language with the help of the constraints epigenetically encoded in our language faculty. On top of that, the individualized I'language can also shift to speaker-external public knowledge when more than one individual having such chunks of I'-knowledge share, speak, and understand the same language. So analogously, there occurs a continual shift to externalized form of intersubjective knowledge of language from the forms of I'-linguistic knowledge when individuals access, create, share, mold and change the intersubjective speakerexternal knowledge of language as they use language. This is how internalized knowledge of language functions inside-out to extend seamlessly into the space of the intersubjective speaker-external knowledge of language. Hence I'-language extends into the speaker-external knowledge space through individuals using language. And this is more possibly due to mind's computations spreading out into the world (Clark 1997). To elaborate on it a little, the point to focus on is that symbolic representations in the architecture in Fig. 1 above are processed independent of considerations of context or culture-based variations in that such symbolic representations have 'non-derived' or 'intrinsic' content (Adams and Aizawa 2010). They have non-derived content as there are some naturalistic conditions on the meanings the symbolic representations in the architecture have and such conditions are respected and fulfilled by the constraints on the architecture in Fig. 1 above (which determine how representations are shipped and mapped into and out of domains -syntax, semantics, phonology etc.). When individuals use language, communicate with each other, such non-derived content of the symbolic representations comes to have derived meanings which come into being by virtue of the intentional acts of individuals using language. Such a transformation of the content of the symbolic representations occurs because of the inside-out extension and shift of internalized knowledge of language into externalized knowledge, and this is how neural processing of linguistic forms allows us to extrapolate external knowledge inside-out. Such outside-in and inside-out shifts between internalized knowledge of language and externalized or speaker-external knowledge can be called undulation. The undulation is elastic because of continuous extension and contraction in the entire knowledge space encompassing both I'-forms of knowledge of language and speaker-external knowledge of language. To be clearer, such undulation occurs only at the ontological level. It is at the ontological level in that epistemology of language presupposes ontology of language so without there being anything called language we cannot talk about knowledge of language. But it is not at the phenomenological level in that the undulation has little to do with the way we become immersed in the experiential stream of the community code of language when we use and communicate in language. Even if meanings are created, 
changed, extended in an experiential space of our living, our experience often misleads us into positing two worlds of knowledge (of language)- one outside the mind and another inside the mind.

Hence there is a continual elastic undulation between I'-knowledge of language and externalized knowledge of language in patterns of complementarity. Such complementarity may well be called an example of parallel complementarity (for details, see Goudsmit 2009) which means that two complementary modes can exist in parallel but description of one cannot be reduced to the other and vice versa. The same can be said about I-linguistic knowledge and externalized intersubjective linguistic knowledge both of which may exist in parallel but description of Ilanguage or I'-language cannot be reduced to externalized intersubjective linguistic knowledge and vice versa. Perhaps that is why Chomskyan internalism claims that the study of E-language is beyond the object of scientific study (Lohndal and Narita 2009). It is true in the sense that unless we have a deeper understanding of Ilanguage or even I'-language, we can never understand externalized linguistic knowledge as I-language (or I'-language) is a precondition for externalized intersubjective linguistic knowledge while the latter is not a precondition for the former. The former is necessary and sufficient, the latter is not.

\section{Internalism and Externalism: Two Aspects of the Same Phenomenal Reality}

The entire picture can now be recast in the form of a holistic picture. Before we proceed to do that, a few more points can be added about the larger reality in which the picture drawn so far can be grounded. Recently Christiansen and Chater (2008) have argued that language structure is shaped and constrained by the nature of our mental representational capacities, socio-pragmatic contexts of language use, cognitive and perceptual constraints. Similarly, Hawkins (2004) has also made a strong case that typological universals of language can be better explained by means of processing constraints on our language processing machinery. This largely corresponds with Dowman et al. (2006) who, pursuing a similar line of argument, has shown in simulation studies that language structure can emerge through iterated learning by virtue of a triadic interaction of individual development, evolution and culture. Again, in a recent article Evans and Levinson (2009) have argued for a coevolutionary model of language within which shared socio-cultural structures underlying language are configured by biological, cognitive and functional constraints. In all, Fitch (2009) has also described such developments as being in the process of tighter articulation and formulation. Assuming that it is really possible for linguistic structures to be shaped by biological, functional, socio-cultural and cognitive constraints, we can now say that the elastic undulation between I'language and externalized intersubjective linguistic knowledge does not occur in a vacuum. It always occurs in an ecological niche of biological, functional, sociocultural and cognitive constraints which partially modulate and shape, rather than determine, I'-language and externalized intersubjective linguistic knowledge and the elastic undulation between them. If we want to account for why language structures are shaped by those constraints, a biological theory must be able to have an answer to that. The reason for this is the amount of research in neurobiological mechanisms 
of language which clearly suggests that language processing at the neural level is shared with other kinds and forms of cognitive processing (Bickerton and Szathmary 2009). If that is the case, it clearly indicates that mechanisms underlying language have been co-opted from the machinery underlying other older cognitive capacities. So such cognitive capacities also control, to some degree, the way language unfolds, develops, changes and is used. This may be the reason why theoretical frameworks like Cognitive Grammar (Lakoff 1987; Langacker 1987) have been more focused on the cognitive operations underlying linguistic structures. And for similar reasons, other theoretical frameworks, from a different perspective altogether, like Functional grammars (Givón 1995, 2002, 2005; Dik 1997a, b; Hengeveld 2005) or Systemic Functional Grammar (Halliday 1973, 1985) have emphasized the functional constraints that underpin the patterns found in language structures. Even if these frameworks have often made the divide between internalism and externalism too wide apart by going to extreme poles, their efforts are now being put under empirical tests as we are gradually getting nearer to insights into the cognitive, computational and functional correspondences between language and its interfaces with other external systems (see for discussion, Di Scuillo 2005a, b).

On the whole, from the discussion above it may well follow that biological, cognitive and functional constraints are like constraining forces that constrain the biological hypothesis space of I'-languages and also externalized intersubjective linguistic knowledge including E-languages. We can imagine a spatially spread malleable sheet and a number of threads pulling it from different directions and thereby warping the spatial topology of the sheet. These threads are now comparable to biological, cognitive, functional and cultural constraints, and the sheet is the malleable linguistic knowledge undulating elastically between I'linguistic organization and externalized organization. For the sake of clarity, we can again add that this malleable linguistic knowledge is in no sense metaphysical. It is as real as socio-cultural conventions or even scientific methodology as scientists follow it. One may not observe them in a physical form, but they exist. Now pulling it all together, we can be a little more specific. Let's say that there are roughly three constraining forces which shape and modulate the control space of linguistic knowledge if we, sort of, extrapolate from biological, cognitive, functional and cultural constraints. These are: processing, (mental) representations and function. Let's not get embroiled in the definitional turf war or complexities of that sort. Processing will roughly mean the cognitive mechanisms underlying memory, attention, action, perception etc. Representation refers to mental data structures used, manipulated, molded, and changed for the purpose of processing (see for some interesting discussion, Edelman 2008). And function will include, more specifically, characteristics of the users of language and the purpose of language use (see for details, Kirby 1996). So function in such sense incorporates sociolinguists' concept of register (Fasold 1990) as well. These three constraining forces mold the spatial geometry of the elastic form of linguistic knowledge as it undulates and shifts from I'-language to externalized linguistic knowledge and vice versa. We can visualize it in the form of the Fig. 2 below.

Here the circular curve forms a trajectory in a three dimensional space of function, processing and representation. Only at this point does it appear that one 
Fig. 2 Complementarity between internalism and externalism in its ecological niche $(\mathrm{P}=$ processing, $\mathrm{F}=$ function, $\mathrm{R}=$ representation)

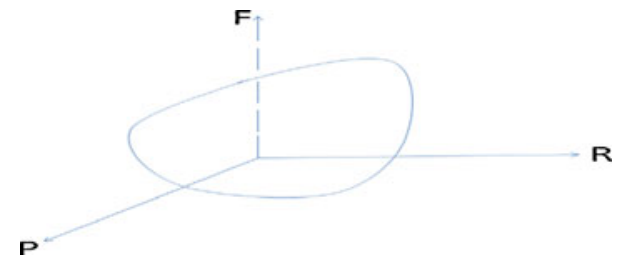

can get a feel of dynamical systems (Edelman 2008) from this figure. Processing, function and representation are like three dimensions or degrees of freedom linguistic knowledge as a whole can afford. At any point of time, linguistic knowledge must be relativized to all three dimensions. As it shifts and moves off closer to processing and representation which are, in some sense, outright individualized, it turns into I'-language or I-language. Analogously, if it stretches out closer towards function, it morphs into externalized intersubjective linguistic knowledge. Or if it extends toward function and processing or toward function and representation, it is in both states simultaneously. The continual undulation between them is modulated by the three dimensions at any point of time. So for example, if we choose any point on the curve of the trajectory in the figure above, that point will correspond to a state of linguistic knowledge whether in I'-state or in externalized state or in some in-between state. Actually, in most cases of language use, we are, in fact, in an in-between state since in parallel complementarity both I'-language and externalized intersubjective linguistic knowledge co-exist, even if the undulation continues. In the figure above, the dimension of function is in dotted lines, mainly because I'-linguistic knowledge can exist independently of externalized intersubjective linguistic knowledge when, for example, there is only one individual user of a language who possesses it. It should be made clear at this point that the epigenetic constraints operating on I'language as specified above in earlier sections are also modulated by the three dimensions of processing, representations and function, but only indirectly by virtue of being encoded in I'-language which is organized from externalized intersubjective linguistic knowledge throughout ontogeny. From this it follows that linguistic knowledge dynamically undulates between I'-language and externalized linguistic knowledge, and with it the components of each also get structured, restructured, changed. That explains why some people often seek explanations of language change in exogenous factors (see for details, Thomsen 2006).

However, the three-dimensional dynamical spatial layout seems to be more pertinent to the requirements of the nature of mental computations as they are fluid, extended, and distributed throughout a network of brain, body, and environment. The space in Fig. 2 is also continuous, so the way mind's distributed computations make a way for the elastic undulation between I'-knowledge and externalized knowledge perfectly fits well with the absence of demarcation in the way function, processing and representation are configured in a three-dimensional space. Again, it should be made clear that this is just a model of how the complementarity between I'-linguistic knowledge and externalized intersubjective linguistic knowledge works in the substrate of three constraining forces. This is not what reality is. But this is what reality may look like. We can note that the 
important insight gained from generative grammar that I-language or competence can be studied independent of processing factors and is at a separate stratum of reality from that of processing is preserved here. In Fig. 2 above, I-language or I'language is only a state in the trajectory when pulled toward processing and representation, but processing and representation are not themselves a part of that state. Rather they bring forth or trigger that state into a level of reality which is separate from the level at which processing and representation operate (see for details, Carr 1990).

In fact, what used to look like clear-cut and simplified cases of internalist or externalist states of linguistic knowledge are actually a part of a vastly complicated scenario, if we view both in terms of parallel complementarity. So internalist and externalist states of linguistic knowledge are in reality aspects of the same phenomenal reality which is dynamic, fluid, labile, ever-shifting, and everundulating. If this breaks apart our familiar picture of linguistic knowledge as fixed in brain or even in mind, let's consider for a moment what this translates into in concrete terms. Maybe the picture presented here is wrong or flawed, but what if it is actually the case? It helps us look into how language really works. Rather than sitting complacent about it, we can go about exploring an enormously convoluted and complicated world where language is in my head and at the same time is a higher plane of existence over and above our subjective confinement. This is not, of course, intended to invite any form of Platonism. That linguistic knowledge undulates between individual space and the supraindividual space is real as evidenced from the existence of E-languages, as Chomskyan linguistics has made a distinction between I-languages and E-languages. A biolinguistic theory that aims at reconciling the two aspects of existential reality about linguistic knowledge may proceed along the lines drawn up over here. No biology of language will ever be complete if we do not resolve the problems revolving around the fundamental concepts about the nature of existence of language and its knowledge.

\section{Implications}

\section{Biological Implications}

Evolvability is one of the criteria that entail many other criteria that constrain theories of language. As Kinsella (2009) has argued, evolvability entails criteria of parsability, neurological instantiability and learnability as well. What this means is that an evolvable system must be parsable, capable of neurological instantiation and learnable by learners- whether children or adults. As Dobzhansky (1973) has stated "nothing in biology makes sense except in the light of evolution", it really requires us to make our biolinguistic theories evolvable. The issue can be touched on from two perspectives. First, the constraints epigenetically encoded in our language faculty operating over the architecture of language have evolved through developmental trajectories which grew consolidated throughout phylogenetic timescales, as has been specified above. This is best captured in an evo-devo scenario (see for details, Locke 2009). Importantly, as we are now aware that the blueprint picture of genome has been abandoned and genes are now viewed as rough 
guidelines as to how and where and what to move (Marcus 2004); so if we say that neither syntax, nor semantics nor even phonology is genetically encoded, what is encoded is the constraints that fix how they interact with one another. This saves us from making any naïve claim that components of language are encoded in genes. This more closely fits with molecular genetics and more generally with modern biology.

Second, the elastic architecture of language with elastic undulation between two states of linguistic knowledge is more viable from a developmental perspective within which we find a lot of granularity in the way developmental disorders affect language (see for details, Fletcher and Miller 2005).

\section{Linguistic Implications}

The elastic architecture of language in Fig. 1 above is sufficiently rich to be able to account for both modularity and distribularity or non-autonomy in language. This may have the desired effect that the huge gap between autonomous generative linguistics (Chomsky 1995) and cognitive linguistics (Lakoff 1987; Langacker 1987) can perhaps be bridged. Maybe at some level claims of both sides have some truth as the architecture of language sketched above may have us believe. On the other hand, the elastic architecture with the notion of elasticity in the nature of linguistic knowledge itself can make us abandon a simplistic picture of the language faculty as fixed, and non-fluid. So under the emerging picture, it becomes increasingly clear that there is no absolute architectural configuration of our language faculty; rather the nature of our language faculty is relative to the linguistic phenomena in a specific language in question. There are many possible configurations of the language faculty. Different versions of generative grammar may well correspond to different configurations of the elastic architecture of language faculty. Hence, Jackendoff's Parallel Architecture (2002) may also be a configuration state of the architecture of language as specified above. Moreover, elasticity in the fabric of knowledge of language itself should not be taken as a criticism of the existing theories of grammar that consider the architecture of grammar as fixed. Rather this should be taken as meaning that there exist many possible architectural configurations of grammar hitherto unknown in a hypothetical hyperspace out of which languages tend to choose from.

And thus linguistic knowledge is neither within the confinements of the skull nor even in the intersubjective realm; rather it is in both, but only in (parallel) complementarity. The continual undulation between these two realms is a capacity which gives Homo Sapiens the unique capability to switch between two states of knowledge thereby making it possible for children to acquire human language.

\section{Cognitive Implications}

From a cognitive perspective, the fact that epigenetically encoded linguistic constraints organize the unorganized external intersubjective linguistic knowledge into I'-forms of knowledge is something very significant as it underpins the organizing capacities of our cognition. That is the reason why Torey (2009) has argued that human mind is actually negative-entropy making system, that is, it 
creates order out of disorder. In this context, he has also speculated upon possible connections to human mind's power to trigger decoherence in quantum phenomena. If this is so, it makes us seriously investigate the cognitive underpinnings of such constraints, and how they are really encoded in a kind of epigenetic synthesis with our genome in concert with the unfolding environmental exposure. Degradation of such constraints will corrode the elasticity of the linguistic knowledge as it shifts between individualized forms and externalized forms of language. More interestingly, the possibility of processing, representational and functional capacities of human beings in pulling around the form of linguistic knowledge as a whole paves the way for the rest of cognition to be related to the core of language faculty. And an approach that readily allows one to relate language to our cognitive capacities, although more indirectly, will perhaps be favored as a biolinguistic theory over the one that does not. This squares up well with the minimalist architecture where the core language machinery (FLN) has only interfaces to Conceptual-Intentional systems and Articulatory-Perceptual systems at the periphery as part of the faculty of language in a broader sense (FLB). And on the other hand, it also tunes in well with the recent forays into the way biological, functional and cognitive constraints modulate language structure (Christiansen and Chater 2008).

\section{Philosophical Implications}

At last, some rudimentary speculations about the philosophical implications of the entire scenario delineated above can be thrown up. The switching over between two epistemological states may be an anathema to some philosophical schools of science like logical positivism, as neither the switch-over nor the any of the states is observable. It is not even justifiable at all. So how can one justify that his/her I- (or I'-) linguistic knowledge switches over, shifts to externalized linguistic knowledge by going beyond the confinements of his/her body including his/her brain? It might be called Platonistic fallacy. But let's note that a lot of research into E-language especially in sociolinguistics, socio-cultural models of language (see for details, Tomasello 2008) clearly shows that E-linguistic phenomena exist. Overall, it requires us to seriously reconsider the notions of fixed epistemology.

\section{Problems, Challenges Ahead}

It is necessary that for a unified biolinguistic approach to be viable, it has to cross a plethora of barriers both theoretical (or conceptual) and empirical. It is highly unlikely that all such problems and challenges will be overcome in a while, but the fact that they exist can make us explore more deeply the nature of language and its biological basis.

- A unified approach to a biolinguistic conception of language will not just be able to reconcile internalism and externalism (or non-internalism) existing in the nature of language. In addition, it has to be capable of being integrated from top to bottom in terms of the three levels- computational, algorithmic and implementational, as postulated by Marr (1982). The implementational level is 
far beyond our reach right now given the present state of our knowledge (Fitch 2009). From the viewpoint of the architecture above in Fig. 1, different kinds of computational primitives as enumerated by Fitch (2009) are realized at the algorithmic level as elastic warping of the architecture in a differential manner for the requirement of the computations involved in linguistic phenomena.

- What the elastic switch-over and shifts between two states of linguistic knowledge- one private and the other public- translate into in concrete terms has not been reduced to precisely detailed specifications. This is not because of lack of interest in doing so. Instead, it is owing to the inherent complexity in the way knowledge of language grows, functions and exists. That is why decades of research in linguistics have not yet been able to tap the exact nature of our competence.

- It is not clear how one would try to relate the complementarity at the level of architecture of language to the complementarity at the higher level of linguistic knowledge as a whole. And whether one transmutes into the other or percolates down or up is quite unknown. One possibility is that the higher level complementarity percolates down to the level of language faculty, since for individual knowledge of language to be useful and functioning, intersubjcetive knowledge is also vital even if there may be a time lag on an evolutionary scale between the first appearance of individual knowledge and that of supraindividual knowledge. But both forms of knowledge may have to go together.

- Another challenge for a unified approach to a biolinguistic conception of language would be to give a precisely detailed, empirically viable and conceptually motivated account of language acquisition. Although we have not yet achieved this, current directions are promising enough. Parameter setting from the exposure to inputs would constitute the building process of language specific rules and constraints for the content of different components of grammar. But such parameter setting has to be guided along the experiential trajectory. The epigenetically encoded constraints operating on the faculty of language can do this job. They are like a cluster of algorithms which are content-independent (while parameters are content-dependent as there are syntactic, phonological and perhaps semantic parameters (Smith and Law 2009)) and which, upon exposure to linguistic inputs, orchestrate the process of parameter setting in a manner faithful to the language in the environment. Children cannot do the job of parameter setting on their own just on the basis of exposure to the language in question, the computational task of parameter setting has to be done by satisfying the requirements of algorithmic (genetic- more correctly, epigenetically encoded) constraints on the faculty of language. More specifically it is the process of parameter setting that actually consists in the gradual organization of externalized intersubjective linguistic knowledge into I'-forms.

\section{Conclusion}

Overall, internalism broadly construed is never at odds with non-internalism or externalism- both are in fact two faces of the same phenomenon. Complementary 
switch-over between them is perhaps the kind of interactionsim that Carr (1990) has intended to mean. The complementarity between I'-forms of knowledge and E-forms of knowledge raises more questions than it answers. Why does it occur the way it does? Is it because of the way language has evolved and develops or due to some other factors? We do not really know. Some speculative answers have already been provided in earlier sections, but these are sketchy enough. Still it is believed that linguistic ontology and its epistemology are more closely coupled than we may imagine them to be. Questions about the nature of language inform questions regarding the form of knowledge language yields to. Such questions are not just philosophical; they constitute the core of the biological basis of language. Therefore, the hope is that ultimately a unified biolinguistic view of language can be formulated on the principles outlined here with all the ingredients fully incorporated and fully fleshed out.

\section{References}

Adams, F., \& Aizawa, K. (2010). Defending the bounds of cognition. In R. Menary (Ed.), The extended mind. Cambridge: MIT.

Archangeli, D., \& Pulleyblank, D. (1994). Grounded phonology. Cambridge: MIT.

Balari, S., \& Lorenzo, G. (2009). Computational phenotypes: where the theory of computation meets evodevo. Biolinguistics, 3(1), 2-60.

Barbieri, M. (2010). On the origin of language: a bridge between biolinguistics and biosemiotics. Biosemiotics, 3, 201-223.

Bates, E. (1994). Modularity, domain specificity and the development of language. Discussions in Neuroscience, $10(1 / 2), 136-149$.

Bates, E. (1997). Origins of language disorders: A comparative approach. In D. J. Thal \& J. Reilly (Eds.), Special issue on origins of communication disorders. Developmental Neuropsychology 13(3), 447476.

Bates, E. (1999). Plasticity, localization and language development. In S. H. Broman \& J. M. Fletcher (Eds.), The changing nervous system: Neurobehavioral consequences of early brain disorders (pp. 214-253). New York: Oxford University Press.

Bickerton, D., \& Szathmary, E. (Eds.). (2009). Biological foundations and origin of syntax. Cambridge: MIT.

Carr, P. (1990). Linguistic realities. New York: Cambridge University Press.

Chomsky, N. (1986). Knowledge of language: Its nature, origin, and use. New York: Praeger.

Chomsky, N. (1995). The minimalist program. Cambridge: MIT.

Chomsky, N. (2000). New horizons in the study of language and mind. Cambridge: Cambridge University Press.

Clark, A. (1997). Being there: Putting brain, body and world together again. Cambridge: MIT.

Christiansen, M. H., \& Chater, N. (2008). Language as shaped by the brain. Behavioral and Brain Sciences, 31(5), 489-509.

Dabrowska, E. (2004). Language, mind and brain. Edinburgh: Edinburgh University Press.

Diamond, A. (2002). Normal development of prefrontal cortex from birth to young adulthood: Cognitive functions, anatomy and biochemistry. In D. T. Stuss \& R. T. Knight (Eds.), Principles of frontal lobe function (pp. 466-503). Oxford: Oxford University Press.

Diamond, A. (2007). Interrelated and interdependent. Developmental Science, 10, 152-158.

Diamond, A. (2009). All or none hypothesis: a global-default mode that characterizes the brain and mind. Developmental Psychology, 45, 130-138.

Di Sciullo, A.-M. (2005a). Asymmetry in morphology. Cambridge: MIT.

Di Sciullo, A.-M. (Ed.). (2005b). UG and external systems: Language, brain and computation. Amsterdam: John Benjamins.

Dik, S. C. (1997a). The Theory of functional grammar. Part I: The structure of the clause. K. Hengeveld (Ed.). Berlin and New York: Mouton de Gruyter. 
Dik, S. C. (1997b). The theory of functional grammar. Part II: Complex and derived structures. K. Hengeveld (Ed.). Berlin and New York: Mouton de Gruyter.

Dobzhansky, T. (1973). Nothing in biology makes sense except in the light of evolution. American Biology Teacher, 35, 125-129.

Dowman, M., Kirby, S., \& Griffiths, T. L. (2006). Innateness and culture in the evolution of language. In A. Cangelosi, A. Smith \& K. Smith (Eds.), Proceedings of the 6th international conference on the evolution of language. World Scientific.

Downing, P., Chan, A. W., Peelan, M., Dodds, C., \& Kanwisher, N. (2005). Domain specificity in visual cortex. Cerebral Cortex, 16, 1453-1461.

Edelman, S. (2008). Computing the mind: How the mind really works. New York: Oxford University Press.

Evans, N., \& Levinson, S. C. (2009). The myth of language universals. Language diversity and its importance for cognitive science. Behavioral and Brain Sciences, 32, 429-492.

Fasold, R. (1990). The sociolinguistics of language. Oxford: Blackwell.

Fitch, W. T. (2009). Prolegomena to a future science of biolinguistics. Biolinguistics, 3(4), 283-320.

Fletcher, P., \& Miller, J. F. (Eds.). (2005). Developmental theory and language disorders. Amsterdam: John Benjamins.

Fodor, J. (1983). The modularity of mind: An essay on faculty psychology. Cambridge: MIT.

Gillon, B. S. (2008). On the semantics/pragmatics distinction. Synthese, 165(3), 373-384.

Givón, T. (1995). Functionalism and grammar. Amsterdam: John Benjamins.

Givón, T. (2002). Bio-linguistics. Amsterdam: John Benjamins.

Givón, T. (2005). Context as other minds: The pragmatics of sociality, cognition and communication. Amsterdam: John Benjamins.

Gobet, F. (2005). Modularity and chunking. In W. Callebaut \& D. Rasskin-Gutman (Eds.), Modularity: Understanding the development and evolution of natural complex systems (pp. 331-352). Cambridge: MIT.

Goudsmit, A. L. (2009). Sense and self-referentiality in living beings. Biosemiotics, 2, 39-46.

Halliday, M. A. K. (1973). Explorations in the functions of language. London: Edwin Arnold.

Halliday, M. A. K. (1985). An introduction to functional grammar. London: Edwin Arnold.

Hauser, M. D., Chomsky, N., \& Fitch, T. W. (2002). The faculty of language: what is it, who has it, and how did it evolve? Science, 298, 1569-1579.

Hawkins, J. A. (2004). Complexity and efficiency in grammars. New York: Oxford University Press.

Hengeveld, K. (2005). Dynamic expression in functional discourse grammar. In C. de Groot \& K. Hengeveld (Eds.), Morphosyntactic expression in functional grammar (pp. 53-86). Berlin and New York: Mouton de Gruyter.

Hengeveld, K., Rijkhoff, J., \& Siewierska, A. (2004). Parts of speech systems and word order. Journal of Linguistics, 40(3), 527-570.

Jackendoff, R. (2002). Foundations of language: Brain, meaning, grammar, evolution. New York: Oxford University Press.

Jackendoff, R. (2007). Language, consciousness, culture: Essays on mental structure. Cambridge: MIT.

Johnson, M. H., \& Morton, J. (1991). Biology and cognitive development: The case of face recognition. Oxford: Blackwell.

Johnson, M. H., Munakata, Y., \& Gilmore, R. O. (Eds.). (1993). Brain development and cognition: A reader. Oxford: Blackwell.

Johnson, M. H., Grossmann, T., \& Cohen-Kadosh, K. (2009). Mapping functional brain development: building a social brain through interactive specialization. Developmental Psychology, 45, 151-155.

Karmiloff-Smith, A. (1992). Beyond modularity: A developmental perspective on cognitive science. Cambridge: MIT.

Karmiloff-Smith, A. (1998). Development itself is the key to understanding developmental disorders. Trends in Cognitive Sciences, 2(10), 389-398.

Karmiloff-Smith, A. (2006). Modularity of mind and language. In K. Brown (Ed.), Encyclopedia of language and linguistics (Vol. 8, pp. 218-224). Oxford: Elsvier.

Kelso, J. A. S. (1995). Dynamic patterns. Cambridge: MIT.

Kelso, J. A. S. (2002). The complementary nature of coordination dynamics: self-organization and the origins of agency. Journal of Nonlinear Phenomena in Complex Systems, 5, 364-371.

Kelso, J. A. S., \& Engstrom, D. A. (2006). The complementary nature. Cambridge: MIT.

Kingsbury, M. A., \& Finlay, B. L. (2001). The cortex in multidimensional space: where do cortical areas come from? Developmental Science, 4, 125-156.

Kinsella, A. R. (2009). Language evolution and syntactic theory. New York: Cambridge University Press. 
Kirby, S. (1996). Function, selection and innateness: The emergence of language universals. Dissertation, University of Edinburgh.

Koster, J. (2009). Ceaseless, unpredictable creativity: language as technology. Biolinguistics, 3(1), 61-92.

Lakoff, G. (1987). Women, fire and dangerous things: What categories tell us about the human mind. Chicago: University of Chicago Press.

Langacker, R. (1987). Foundations of cognitive grammar (Vol. 2). Stanford: Stanford University Press.

Locke, J. L. (2009). Evolutionary developmental linguistics: naturalization of the faculty of language. Language Sciences, 31, 33-59.

Lohndal, T., \& Narita, H. (2009). Internalism as methodology. Biolinguistics, 3(4), 321-331.

Marcus, G. (2004). The birth of the mind: How a tiny number of genes creates the complexities of human thought. New York: Basic Books.

Marr, D. (1982). Vision: A computational investigation into the human representation and processing of visual information. New York: W. H. Freeman.

Mascaró, J. (2007). External allomorphy and lexical representation. Linguistic Inquiry, 38, 715-735.

Mondal, P. (2009). How language processing constrains (computational) natural language processing: A cognitive perspective. In Proceedings of 23rd Pacific Asia Conference on Language, Information and Computation. Hong Kong.

Neelman, A., \& Weerman, F. (1998). Flexible syntax: A theory of case and arguments. Dordrecht: Kluwer. Smith, N., \& Law, A. (2009). On parametric (and non-parametric) variation. Biolinguistics, 3(4), 332-343.

Thomsen, N. O. (Ed.). (2006). Competing models of linguistic change: Evolution and beyond (Current Issues in Linguistic Theory 279). Amsterdam: John Benjamins.

Tomasello, M. (2008). The origins of human communication. Cambridge: MIT.

Torey, Z. (2009). The crucible of consciousness: An integrated theory of mind and brain. Cambridge: MIT. 